\title{
Desenvolvimento inicial e nutrição de mudas de mogno-africano em resposta à adubação nitrogenada e fosfatada
}

\author{
Development and nutrition of African mahogany seedlings in response to \\ nitrogen and phosphorus fertilization
}

\author{
Matheus da Silva Araújo ${ }^{1}$ (1), Stephany Diolino Cunha² (B), Alcides Gatto ${ }^{3}$ (B), \\ Bárbara Elias Reis Hodecker ${ }^{3}$ (1) , Adilson Pelá4 (1) \\ 1Escola Superior de Agricultura "Luiz de Queiroz" - ESALQ, Universidade de São Paulo - USP, Piracicaba, SP, Brasil \\ ${ }^{2}$ Universidade Federal de Lavras - UFLA, Lavras, MG, Brasil \\ ${ }^{3}$ Universidade de Brasília - UnB, Brasília, DF, Brasil \\ ${ }^{4}$ Universidade Estadual de Goiás - UEG, Ipameri, GO, Brasil
}

Como citar: Araújo, M. S., Cunha, S. D., Gatto, A., Hodecker, B. E. R., \& Pelá, A. (2020). Desenvolvimento inicial e nutrição de mudas de mogno-africano em resposta à adubação nitrogenada e fosfatada. Scientia Forestalis, 48(125), e2938. https://doi.org/10.18671/scifor.v48n125.14

\begin{abstract}
Resumo
O mogno-africano é uma espécie com grande potencial econômico para o setor florestal brasileiro, no entanto, carece de informações relacionadas às suas exigências nutricionais, principalmente na fase de muda. Objetivou-se avaliar o efeito de doses de nitrogênio (N) e fósforo (P) no desenvolvimento inicial e nutrição de mudas de mogno-africano. $O$ experimento foi realizado em vasos com capacidade de $5 \mathrm{dm}^{3}$. O delineamento experimental utilizado foi o inteiramente casualizado, em esquema fatorial $5 \times 5$, com quatro repetições. Os tratamentos foram constituídos de cinco doses de $\mathrm{N}$ e cinco doses de $\mathrm{P}: 0$, 50, 100, 150 e $200 \mathrm{mg}^{-\mathrm{dm}^{-3}}$. Aos 120 dias após transplantio, foram analisadas: a altura da planta $(\mathrm{H}), \mathrm{O}$ diâmetro do coleto (DC), o número de folíolos (NF), a massa de matéria seca da parte aérea (MSPA), a massa de matéria seca de raiz (MSR), a massa de matéria seca total (MST), os teores de $\mathrm{N}$ e $\mathrm{P}$ nas folhas (TNF e TPF), no caule (TNC e TPC) e nas raízes (TNR e TPR). A aplicação de $200 \mathrm{mg}^{-\mathrm{dm}^{-3}} \mathrm{de} \mathrm{P}$ associada à adição de $100{\mathrm{mg} . \mathrm{dm}^{-3}}^{-3}$ de $\mathrm{N}$ proporcionaram o máximo crescimento nas mudas de mogno-africano. O N e o $\mathrm{P}$ possuem papel fundamental no desenvolvimento da espécie, uma vez que são responsáveis por processos que fomentam as atividades celulares e fotossintéticas proporcionando assim, o crescimento das mudas. Deste modo, os incrementos observados nas mudas de mogno-africano em razão do aumento das doses de $\mathrm{N}$ e $\mathrm{P}$ evidenciam a importância da adequada nutrição nitrogenada e fosfatada na produção de mudas da espécie.
\end{abstract}

Palavras-chave: Crescimento; Macronutrientes; Nutrição mineral; Produção de mudas; Silvicultura.

\begin{abstract}
The African mahogany species has great economic potential for the Brazilian forest sector, however it is still not clear its specific nutritional requirements, mainly during the seedling stage. The objective of this work was to evaluate the effect of doses of nitrogen $(N)$ and phosphorus $(P)$ in the initial development and nutrition of African mahogany seedlings. The experiment was performed in pots with capacity of $5 \mathrm{dm}^{3}$. The experimental design used was the completely randomized design in $5 \times 5$ factorial scheme, with four replicates. The treatments were consisted by five doses of $\mathrm{N}$, combined with five doses of $\mathrm{P}:$ : $, 50,100,150$ and $200 \mathrm{mg}^{-\mathrm{dm}^{-3}} .120$ days after transplanting, the seedlings were collected and then analyzed: the plant height (H), diameter of collect (DC), number of leaves (NF), shoot dry matter (MSPA), root dry matter (MSR), total dry matter (MST), concentration of N and P in leaves (TNF and TPF) stem
\end{abstract}

Fonte de financiamento: Universidade de Brasília e a Coordenação de Aperfeiçoamento de Pessoal de Nível Superior (CAPES).

Conflito de interesse: Nada a declarar.

Autor correspondente: araujomatheus@usp.br

Recebido: 24 junho 2018

Aceito: 6 abril 2019.

Editor: Francides Gomes da Silva Júnior.

(i) Este é um artigo publicado em acesso aberto (Open Access) sob a licença Creative Commons Attribution, que permite uso, distribuição e reprodução cc) em qualquer meio, sem restrições desde que o trabalho original seja corretamente citado. 
(TNC and TPC) and root ES (TNR and TPR). The application of $200 \mathrm{mg} \cdot \mathrm{dm}^{-3}$ of P associated with the addition of $100 \mathrm{mg} . \mathrm{dm}^{-3}$ of $\mathrm{N}$ provided the most increment in growth of African mahogany seedlings. $\mathrm{N}$ and $\mathrm{P}$ are nutrients, which play important roles in the plant development, since their functions are related to the processes that regulate cellular and photosynthetic activities, thus providing the seedling growth. The greater African mahogany seedlings development due to increasing doses of $\mathrm{N}$ and $\mathrm{P}$ emphasizes the importance of $\mathrm{N}$ and $\mathrm{P}$ supply during seedling productions.

Keywords: Growth; Macronutrients; Mineral nutrition; Seedling production; Forestry.

\section{INTRODUÇÃO}

Dentre as espécies utilizadas para fins de produção de madeira nobre no mercado internacional destaca-se o mogno-africano (Khaya ivorensis A. Chev). Esta espécie pertence à família Meliaceae, que possui ocorrência natural da África ocidental, em especial na Costa do Marfim, Gana, Togo, Benin, Nigéria, sul de Camarões até a província de Cabinda (Danquah et al., 2011). Sua madeira apresenta alto valor comercial no mercado internacional, sendo utilizada principalmente em laminação, movelaria de luxo, nas construções de interiores e construção naval (Pinheiro et al., 2011).

No Brasil, a espécie está ganhando destaque pelo fato de ser resistente ao ataque e danos provocados por Hypsipyla grandella (Zeller), praga que causa severos danos aos plantios do mogno nativo (Swietenia macrophylla King) (Pinheiro et al., 2013). Além disso, o mogno-africano ( $K$. ivorensis A. Chev) apresenta boa adaptabilidade aos solos brasileiros e ótimas características silviculturais (Verzignassi et al., 2009), como fuste retilíneo, ausência de bifurcações e baixos índices de infestação de pragas e doenças, características que favorecem o desenvolvimento, com produtividade média de $400 \mathrm{~m}^{3}$.ha-1 em ciclo de 21 anos (Ribeiro et al., 2017).

A adoção de práticas adequadas de manejo em viveiros e a campo são importantes para a produção de mudas de boa qualidade e estabelecimento da cultura no campo (Ryan et al., 2010). Dentre as práticas, o manejo eficiente da nutrição mineral em fase de viveiro e da fertilidade do solo são fatores determinantes na produtividade das espécies florestais, principalmente na fase inicial de desenvolvimento (Cardoso et al., 2015). A adequada produção de mudas constitui importante etapa para obtenção de plantas vigorosas em campo, além de assegurar crescimento satisfatório, permitindo desse modo, maior sucesso no estabelecimento da cultura (Leal et al., 2013).

Dentre os elementos essenciais, o fósforo $(P)$ é o macronutriente mais aplicado nos cultivos de espécies florestais. Principalmente nos estádios iniciais de germinação, produção de mudas e estabelecimento no campo, e entre todos os demais é o que apresenta maior deficiência nos solos tropicais, como os solos de Cerrado (Cabral et al., 2016), devido às interações entre as argilas não silicatadas, em especial os óxidos de Fe e Al a este elemento (Novais et al., 2007).

Outro macronutriente importante no crescimento inicial das mudas é o nitrogênio (N), responsável por compor as principais moléculas e estruturas da planta, como clorofila, ácidos nucleicos, proteínas, dentre outros. O N é altamente móvel na planta e translocado entre tecidos velhos para a formação de novas estruturas (Malavolta et al., 1997; Marschner, 2012). De modo que pesquisadores já relataram que a deficiência deste nutriente influencia processos vitais na planta, comprometendo as taxas fotossintéticas e afetando diretamente o crescimento (Bissani et al., 2004).

Devido à escassez de informações relacionadas à exigência nutricional do mogno-africano quanto à nutrição nitrogenada e fosfatada (Silva et al., 2016) e seu potencial econômico e, a fim de aumentar a produção de mudas de boa qualidade (nutricional e fisiológica), faz-se necessário o desenvolvimento de estudos detalhados sobre o fornecimento e doses adequadas destes nutrientes para o crescimento inicial da espécie.

Diante do exposto nosso objetivo foi avaliar o efeito de doses de nitrogênio e fósforo no desenvolvimento inicial e na nutrição de mudas de mogno-africano (Khaya ivorensis A. Chev) em condições de viveiro. 


\section{MATERIAL E MÉTODOS}

\section{Condições experimentais}

O experimento foi conduzido no Viveiro de Pesquisas, em área experimental da

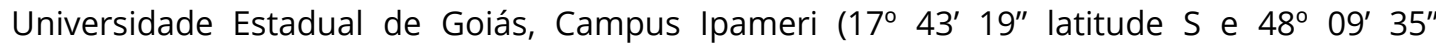
longitude W; e altitude de $764 \mathrm{~m}$ ), no período de agosto a dezembro de 2016. No período experimental as temperaturas dentro do viveiro variaram entre $10^{\circ} \mathrm{C}$ (mínima) e $44^{\circ} \mathrm{C}$ (máxima) e a média foi de $29^{\circ} \mathrm{C}$.

A espécie utilizada de mogno-africano foi o Khaya ivorensis A. Chev, e as mudas foram adquiridas com 120 dias de idade, em tubetes com capacidade de $290 \mathrm{~cm}^{3}$, preenchidos com

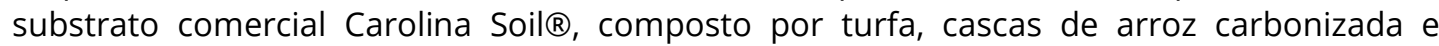
vermiculita. Durante esse período, as mudas não receberam nenhuma adubação mineral.

Como substrato, utilizou-se um Latossolo Vermelho-Amarelo distrófico (Empresa Brasileira de Pesquisa Agropecuária, 2013), coletado na camada subsuperficial $(0,40 \mathrm{~m})$, seco ao ar e posteriormente peneirado e caracterizado quimicamente (Raij et al., 2001) (Tabela 1).

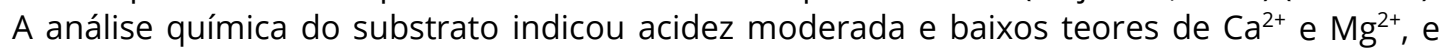
para sua correção foi aplicado calcário dolomítico PRNT 92, visando elevar a saturação por bases para 60\% (Ribeiro et al., 1999). Durante 45 dias, as amostras de solo foram incubadas e a umidade do substrato mantido à $60 \%$ da capacidade máxima de retenção de água do solo.

Tabela 1. Características químicas do substrato para produção de mudas de mogno-africano.

\begin{tabular}{|c|c|c|c|c|c|c|c|c|}
\hline $\mathrm{pH}$ & $\mathbf{P}$ & M.O. & $\mathbf{K}^{+}$ & $\mathrm{Ca}^{2+}$ & $\mathrm{Mg}^{2+}$ & $\mathrm{H}+\mathrm{Al}$ & CTC & V \\
\hline $\mathrm{CaCl}_{2}$ & mg.dm-3 & g.dm $\mathrm{dm}^{-3}$ & \multicolumn{5}{|c|}{ 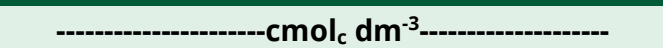 } & $\%$ \\
\hline 5,4 & 0,8 & 7,0 & 0,04 & 0,2 & 0,1 & 1,8 & 2,14 & 16,09 \\
\hline \multicolumn{2}{|r|}{ B } & \multicolumn{2}{|c|}{$\mathrm{Cu}$} & \multicolumn{2}{|c|}{$\mathrm{Fe}$} & \multicolumn{2}{|c|}{ Mn } & $\mathrm{Zn}$ \\
\hline \multicolumn{9}{|c|}{ 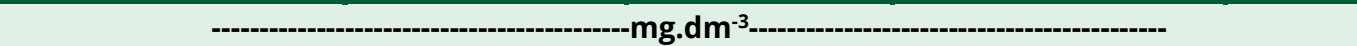 } \\
\hline \multicolumn{2}{|r|}{0,28} & \multicolumn{2}{|c|}{0,3} & \multicolumn{2}{|c|}{34} & \multicolumn{2}{|c|}{10,1} & 0,3 \\
\hline
\end{tabular}

Extratores: $\mathrm{P}$, e K - Mehlich-1; $\mathrm{Ca}^{2+}, \mathrm{Mg}^{2+}$ e $\mathrm{Al}^{3+}-\mathrm{KCl} 1 \mathrm{~mol} / \mathrm{L} ; \mathrm{H}+\mathrm{Al}$ - Acetado de cálcio 0,5 mol/L a pH 7,0; V: saturação por bases (\%).

Após a incubação, o solo recebeu adubação básica de micronutrientes: Mo $\left(0,1 \mathrm{mg} \cdot \mathrm{dm}^{-3}\right), \mathrm{Cu}\left(0,5 \mathrm{mg} \cdot \mathrm{dm}^{-3}\right), B\left(0,5 \mathrm{mg} \cdot \mathrm{dm}^{-3}\right), \mathrm{Zn}\left(5 \mathrm{mg} \cdot \mathrm{dm}^{-3}\right), \mathrm{Mn}\left(1,5 \mathrm{mg} \cdot \mathrm{dm}^{-3}\right)$, e o macronutriente $\mathrm{K}\left(80 \mathrm{mg} \cdot \mathrm{dm}^{-3}\right)$, conforme recomendação proposta por Araújo et al. (2017) para a espécie Khaya senegalensis A Juss. As fontes utilizadas foram: molibdato de sódio, sulfato de cobre, ácido bórico, sulfato de zinco, sulfato de manganês e cloreto de potássio, respectivamente.

Aos 120 dias de idade, as mudas foram transplantadas para vasos de polietileno preto com capacidade de $5 \mathrm{dm}^{3}$. As mudas apresentavam altura média de $29,5 \mathrm{~cm} /$ planta e diâmetro do coleto médio de $7,5 \mathrm{~mm} /$ planta. Para a condução do experimento, foi utilizado o delineamento experimental inteiramente casualizado em esquema fatorial $5 \times 5$, com vinte e cinco tratamentos e quatro repetições cada, totalizando 100 unidades experimentais. Os tratamentos consistiram de cinco doses de nitrogênio (N): 0, 50, 100, 150 e $200 \mathrm{mg}^{-\mathrm{dm}^{-3}}$ combinadas com cinco doses consecutivas de fósforo (P): 0, 50, 100, 150 e $200 \mathrm{mg}^{-\mathrm{dm}^{-3}}$, sendo utilizada a ureia $\left(\mathrm{CH}_{4} \mathrm{~N}_{2} \mathrm{O}\right)$ e o superfosfato triplo $\left(\mathrm{P}_{2} \mathrm{O}_{5}\right)$ como fontes de $\mathrm{N}$ e $\mathrm{P}$, respectivamente.

As doses de $\mathrm{N}$ foram divididas em quatro aplicações $(0,30,60$ e 90 dias após transplantio) para evitar perdas por lixiviação e volatilização, aplicadas via solução. As doses de $P$, foram aplicadas mediante à incorporação ao substrato, em função da baixa mobilidade do fósforo no solo, revolvendo totalmente o mesmo no momento do transplantio, em única aplicação. 
Durante o período de condução do experimento, a umidade do solo foi mantida à $60 \%$ da capacidade máxima de retenção de água no solo. A água evapotranspirada foi reposta diariamente por meio de pesagem aleatória dos vasos. Foi realizado também controle manual de plantas invasoras nos vasos, eliminando-as logo que surgiam.

\section{Análises e características avaliadas}

Após 120 dias do transplantio, as variáveis morfológicas das mudas foram analisadas, por meio da medição de diâmetro do coleto $(\mathrm{mm})$ (DC; utilizando um paquímetro digital); do número de folíolos (NF; por meio de contagem) e da altura das mudas $(\mathrm{cm})(\mathrm{H}$; medido desde o coleto até o ápice com o auxílio de régua graduada).

Em seguida, as plantas foram separadas em parte aérea (folhas e caule) e raízes para a determinação de massa de matéria seca, com uso de tesoura de poda. Para a separação das raízes, junto ao substrato dos vasos, foi utilizada peneira com malha de $4 \mathrm{~mm}$. Por fim, foi realizada a lavagem de cada componente das plantas com água destilada e, posteriormente, as mesmas foram colocadas em estufa de circulação forçada de ar por 72 horas, em temperatura de $70{ }^{\circ} \mathrm{C}$, até a obtenção de massa de matéria seca constante. Após secagem, realizou-se a pesagem dos componentes das plantas em balança analítica com precisão de 0,01 g para determinação da massa de matéria seca da parte aérea (g) (MSPA) e das raízes (g) (MSR), cujos valores foram somados para obtenção da massa de matéria seca total (g) (MST).

Para a análise dos teores de $\mathrm{N}$ e $\mathrm{P}$ nas folhas, caule e raízes $\left(\mathrm{g} \cdot \mathrm{kg}^{-1}\right)$, foram realizadas a moagem, em moinho de aço inoxidável do tipo Willey, com peneira de 20 Mesh. Para a determinação dos teores de N, foi empregado o método de Kjeldahl e, para a determinação dos teores de $\mathrm{P}$, foi utilizado o método de espectrofotometria com azul-de-molibdênio (Silva, 2009).

Verificamos se os parâmetros das mudas (a altura da planta, o diâmetro do coleto, o número de folíolos, a massa de matéria seca da parte aérea, a massa de matéria seca de raiz, a massa de matéria seca total, os teores de $\mathrm{N}$ e $\mathrm{P}$ nas folhas, no caule e nas raízes) se diferiam entre os tratamentos por meio de análise de variância (teste $\mathrm{F}$ ) e teste post hoc de Tukey. Verificamos os pressupostos de homogeneidade de variâncias por meio do teste de Levene e de normalidade por meio do teste de Shapiro-Wilk. Para analisar as influências significativas das doses de $\mathrm{N}$ e P sobre as características avaliadas, trabalhou-se com ajustes das análises de regressão com cortes. Assim os parâmetros que apresentaram ajuste de regressão linear significativo foram apresentados à inclinação da reta. Essas análises estatísticas foram conduzidas utilizando o software Statistica 8.0. O nível de significância empregado em todas as análises foi de $5 \%$ de probabilidade.

\section{RESULTADOS E DISCUSSÃO}

\section{Características morfológicas}

As mudas cultivadas nas doses 0,100 e $150 \mathrm{mg}^{-\mathrm{dm}^{-3}}$ de $\mathrm{N}$ não apresentaram diferenças significativas nas variáveis avaliadas nos diferentes níveis de $P(0 ; 50 ; 100$ e $150 \mathrm{mg} \cdot \mathrm{dm}^{-3}$ ) (Tabela 2). Entretanto, na dose $50 \mathrm{mg} \cdot \mathrm{dm}^{-3}$ de $\mathrm{N}$, as variáveis DC e MSPA apresentaram diferenças entre os níveis de P. De modo que, na dose $50 \mathrm{mg} . \mathrm{dm}^{-3} \mathrm{de} \mathrm{N}$, os tratamentos de 100 e $200 \mathrm{mg} \cdot \mathrm{dm}^{-3}$ de $\mathrm{P}$ apresentaram as maiores médias de DC, entretanto não se diferiram dos tratamentos 50 e $150 \mathrm{mg}^{-\mathrm{dm}^{-3}}$, e foram similares às mudas cultivadas na ausência de $\mathrm{P}\left(0 \mathrm{mg} \cdot \mathrm{dm}^{-3}\right)$. 
Tabela 2. Efeito das doses de nitrogênio $(N)$ e fósforo $(P)$ na altura $(H)$, diâmetro do coleto (DC), número de folíolos (NF), massa de matéria seca parte aérea (MSPA), massa de matéria seca raiz (MSR) e massa de matéria seca total (MST) de mudas de mogno-africano.

\begin{tabular}{|c|c|c|c|c|c|c|c|}
\hline $\mathbf{N}$ & $\mathbf{P}$ & $\mathbf{H}$ & DC & NF & MSPA & MSR & MST \\
\hline \multicolumn{2}{|c|}{ Doses (mg.dm-3) } & $(\mathrm{cm})$ & $(\mathrm{mm})$ & $\left(n^{\circ}\right)$ & \multicolumn{3}{|c|}{ 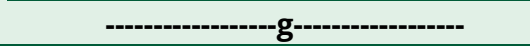 } \\
\hline \multirow{6}{*}{0} & 0 & $32,2 a$ & $10,2 a$ & $21 a$ & $13,7 a$ & $10,7 a$ & $24,2 a$ \\
\hline & 50 & $32,2 a$ & $11,2 \mathrm{a}$ & $29 a$ & $16,2 \mathrm{a}$ & $12,2 \mathrm{a}$ & $29,0 a$ \\
\hline & 100 & $32,7 a$ & $10,7 a$ & $26 a$ & $12,5 a$ & $11,7 a$ & $24,5 a$ \\
\hline & 150 & $36,7 a$ & $10,2 a$ & $27 a$ & $12,5 a$ & $10,2 a$ & $22,5 a$ \\
\hline & 200 & $36,5 a$ & $10,7 a$ & $29 a$ & $16,0 a$ & $11,5 a$ & $27,2 a$ \\
\hline & & $F=1,3 ; p<0,30$ & $F=2,8 ; p<0,06$ & $F=0,3 ; p<0,80$ & $F=1,7 ; p<0,18$ & $F=0,5 ; p<0,73$ & $\begin{array}{l}F=1,4 ; \\
p<0,26\end{array}$ \\
\hline \multirow{5}{*}{50} & 0 & $41,5 a$ & $11,5 b$ & $48 a$ & $25,0 \mathrm{~b}$ & $15,7 a$ & $41,2 a$ \\
\hline & 50 & $43,7 a$ & $12,5 a b$ & $65 a$ & $29,7 a b$ & $15,0 a$ & $44,7 a$ \\
\hline & 100 & $45,0 a$ & $14,2 \mathrm{a}$ & $60 a$ & $35,0 a$ & $19,2 a$ & $54,5 a$ \\
\hline & 150 & $50,5 a$ & $13,5 a b$ & $57 a$ & $31,5 a b$ & $15,2 a$ & $46,5 a$ \\
\hline & 200 & $49,7 a$ & $14,2 \mathrm{a}$ & $57 a$ & $28,7 a b$ & $16,5 a$ & $45,0 a$ \\
\hline \multirow{7}{*}{100} & & $F=0,5 ; p<0,69$ & $F=5,6 ; p<0,00$ & $F=0,7 ; p<0,56$ & $F=4,4 ; p<0,01$ & $F=1,0 ; p<0,43$ & $\begin{array}{l}F=2,5 \\
p<0,08\end{array}$ \\
\hline & 0 & $36,2 a$ & $12,2 \mathrm{a}$ & $53 a$ & $29,7 a$ & $12,7 a$ & $42,2 a$ \\
\hline & 50 & $45,0 a$ & $12,5 a$ & $61 a$ & $30,5 a$ & $12,5 a$ & $42,7 a$ \\
\hline & 100 & $53,5 a$ & $14,0 a$ & $69 a$ & $36,7 a$ & $14,5 a$ & $51,5 a$ \\
\hline & 150 & $50,2 a$ & $13,2 a$ & $62 a$ & $41,0 a$ & $14,2 \mathrm{a}$ & $54,7 a$ \\
\hline & 200 & $55,2 a$ & $14,2 a$ & $64 a$ & $40,2 a$ & $18,5 a$ & $58,5 a$ \\
\hline & & $F=2,5 ; p<0,08$ & $F=2,0 ; p<0,14$ & $F=0,5 ; p<0,74$ & $F=2,0 ; p<0,13$ & $F=1,4 ; p<0,25$ & $\begin{array}{l}F=2,5 \\
p<0,07\end{array}$ \\
\hline \multirow{5}{*}{150} & 0 & $40,5 a$ & $12,5 a$ & $64 a$ & $32,0 a$ & $13,7 a$ & $45,5 a$ \\
\hline & 50 & $41,5 a$ & $12,7 a$ & $74 a$ & $34,2 a$ & $15,2 a$ & $49,5 a$ \\
\hline & 100 & $46,2 a$ & $12,2 a$ & $69 a$ & $37,7 a$ & $16,7 a$ & $54,2 a$ \\
\hline & 150 & $41,2 \mathrm{a}$ & $13,0 a$ & $73 a$ & $43,2 a$ & $19,5 a$ & $62,5 a$ \\
\hline & 200 & $44,7 a$ & $12,5 a$ & $77 a$ & $39,5 a$ & $17,2 \mathrm{a}$ & $56,7 a$ \\
\hline \multirow{7}{*}{200} & & $F=0,3 ; p<0,85$ & $F=0,1 ; p<0,93$ & $F=0,3 ; p<0,83$ & $F=0,9 ; p<0,44$ & $F=0,4 ; p<0,77$ & $\begin{array}{l}F=0,8 ; \\
p<0,53\end{array}$ \\
\hline & 0 & $46,2 a$ & $10,7 a b$ & $60 a$ & $30,0 a$ & $10,2 b$ & $40,2 a$ \\
\hline & 50 & $42,5 a$ & $10,2 b$ & $61 a$ & $27,0 a$ & $8,2 b$ & $35,2 a$ \\
\hline & 100 & $45,0 a$ & $13,0 a$ & $80 a$ & $39,7 a$ & $14,5 a b$ & $54,0 a$ \\
\hline & 150 & $51,7 a$ & $12,7 a$ & $67 a$ & $38,5 a$ & $22,0 a$ & $60,5 a$ \\
\hline & 200 & $51,7 a$ & $13,0 a$ & $85 a$ & $44,0 a$ & $13,0 a b$ & $57,2 a$ \\
\hline & & $F=1,2 ; p<0,32$ & $F=6,6 ; p<0,00$ & $F=1,8 ; p<0,18$ & $F=2,7 ; p<0,12$ & $F=4,4 ; p<0,01$ & $\begin{array}{l}F=2,5 \\
p<0,08\end{array}$ \\
\hline
\end{tabular}

Médias seguidas de mesma letra na coluna não diferem significativamente.

Para a dose de $200 \mathrm{mg} \cdot \mathrm{dm}^{-3}$ de N, o DC foi significativamente superior nas doses entre 100 e $200 \mathrm{mg} \mathrm{dm}^{-3}$ de $P$, em relação aos demais níveis de $P$ (Tabela 2). Para esse mesmo tratamento, observaram-se diferenças na MSR, onde as mudas submetidas à dose $100 \mathrm{mg} \cdot \mathrm{dm}^{-3}$ de $\mathrm{P}$, apresentaram as maiores médias em relação aos tratamentos 0 e $50 \mathrm{mg}^{\mathrm{dm}} \mathrm{dm}^{-3}$, entretanto não se diferiu dos tratamentos 100 e $200 \mathrm{mg} \cdot \mathrm{dm}^{-3} \mathrm{de} P$.

A altura média $(\mathrm{H})$ das mudas de mogno-africano aferida nos indivíduos submetidos à dose de $100 \mathrm{mg} \cdot \mathrm{dm}^{-3}$ de $\mathrm{N}$ e que receberam a dose máxima de $\mathrm{P}\left(200 \mathrm{mg}^{\mathrm{dm}} \mathrm{dm}^{-3}\right)$ foi de $55,3 \mathrm{~cm}$, valor $52,7 \%$ superior à média de altura observada $(36,2 \mathrm{~cm} /$ planta) nos indivíduos que não receberam adubação fosfatada $\left(0 \mathrm{mg} \cdot \mathrm{dm}^{-3}\right)$, entretanto não se diferiram entre si (Tabela 2). Para as mudas que receberam $50 \mathrm{mg}^{-\mathrm{dm}^{-3}}$ de $\mathrm{N}$, a maior média de $\mathrm{H}$ foi de $49,6 \mathrm{~cm} /$ planta, também obtida na dose máxima de $\mathrm{P}\left(200 \mathrm{mg} \cdot \mathrm{dm}^{-3}\right), 19,8 \%$ superior à média de altura observada $(41,4 \mathrm{~cm} /$ planta) nas plantas cultivadas na ausência de P (Tabela 2). Para as demais variáveis, os incrementos obtidos com a maior dose de $\mathrm{P}\left(200 \mathrm{mg} . \mathrm{dm}^{-3}\right)$ em 
relação ao tratamento controle (0 $\mathrm{mg}^{-\mathrm{dm}^{-3}}$ de $\mathrm{P}$ ) foram de: $16,6 \%$ para o $\mathrm{DC} ; 35,2 \%$ para MSPA; 47,15\% para MSR; e 38,5\% MST (Tabela 2).

Nos ajustes de regressão, entre as doses de $\mathrm{P}$, foram observadas associação positiva quando as mudas foram submetidas à $100 \mathrm{mg}^{-\mathrm{dm}^{-3}}$ de $\mathrm{N}$, com incremento das variáveis $\mathrm{H}$, DC, MSPA, MSR e MST (Figura $1 \mathrm{~A}, \mathrm{~B}, \mathrm{D}, \mathrm{E}$ e F) e para $\mathrm{H}$ também quando submetida à dose $50 \mathrm{mg}^{-\mathrm{dm}^{-3}}$ de N (Figura $1 \mathrm{~A}$ ). Para NF, não houve associação significativa com as doses de P para nenhuma das doses de $\mathrm{N}$ (Figura 1C).
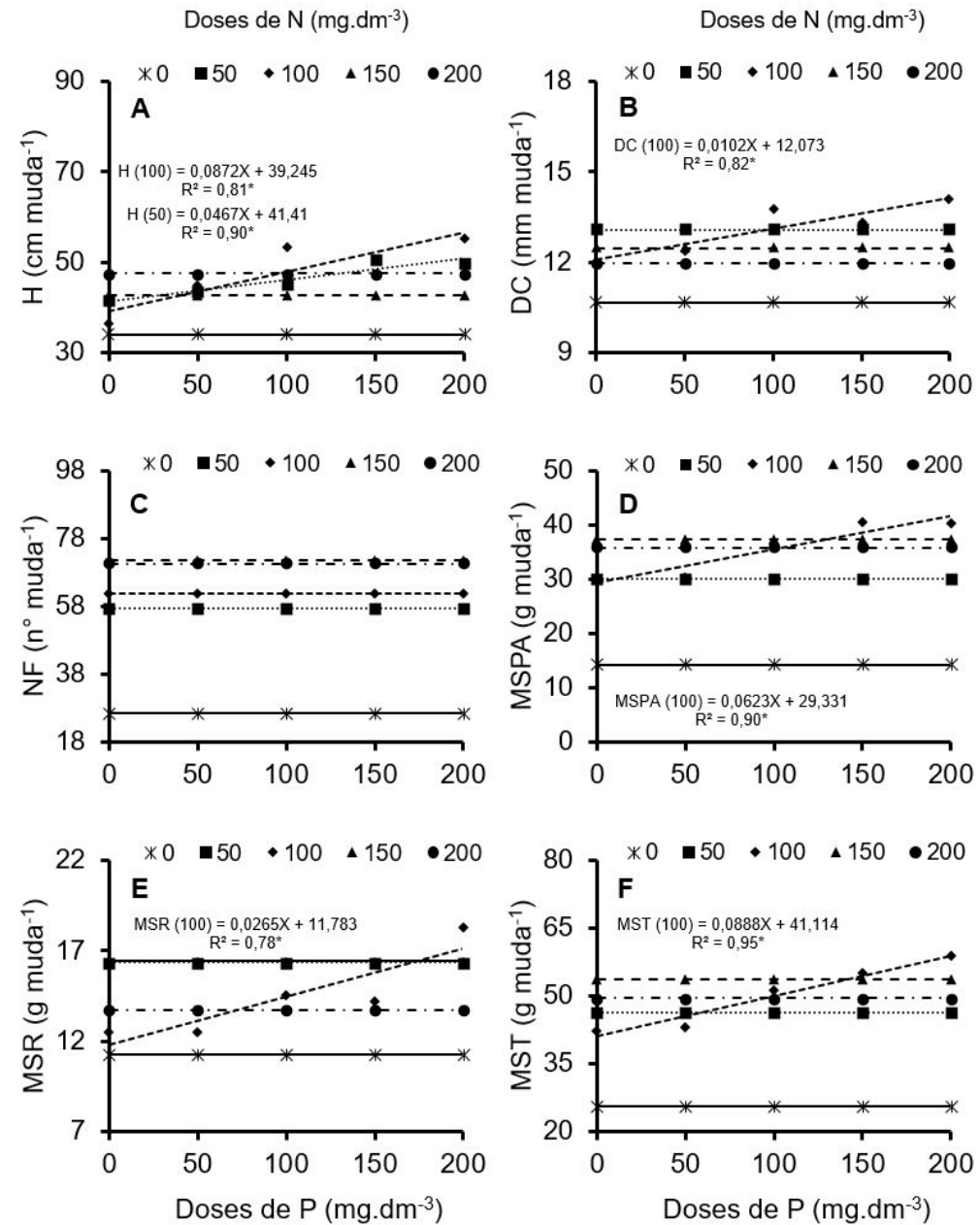

Figura 1. Ajuste de regressão para altura das plantas $(H)(A)$, diâmetro do coleto $(D C)(B)$, número de folíolos (NF) (C), massa de matéria seca parte aérea (MSPA) (D), massa de matéria seca de raiz (MSR) (E) e massa de matéria seca total (MST) (F) de mudas de mogno-africano adubadas com doses de $\mathrm{N}$ e $\mathrm{P}$.

\section{Teor de nutrientes nas mudas}

As mudas cultivadas na ausência da adubação nitrogenada $\left(0 \mathrm{mg}^{-\mathrm{dm}^{-3}}\right.$ de $\left.\mathrm{N}\right)$ apresentaram diferenças estatísticas somente nas variáveis TNF e TPC, obtendo as maiores médias quando submetidas à dose máxima de $\mathrm{P}\left(200 \mathrm{mg}^{-\mathrm{dm}^{-3}}\right)$ (Tabela 3). Na presença da adubação nitrogenada (50 mg. $\left.\mathrm{dm}^{-3}\right)$, as mudas apresentaram diferenças entre os níveis de $\mathrm{P}$ para TPF, TPC e TPR. Na dose $100 \mathrm{mg} \mathrm{dm}^{-3}$ de $\mathrm{N}$, os teores nutricionais sofreram influência dos níveis de P para o TNF, TNC e TPR. Já na dose $150 \mathrm{mg}^{-\mathrm{dm}^{-3}}$ de N, somente o TNC e o TPC apresentaram diferenças estatísticas. Quando as mudas foram submetidas a dose máxima de $\mathrm{N}\left(200 \mathrm{mg} \mathrm{dm}^{-3}\right)$ todos os teores de $\mathrm{N}$ e $\mathrm{P}$ nas folhas, caule e raízes das plantas sofreram influência positiva dos diferentes níveis de $P$, exceto para TPF. 
Tabela 3. Efeito das doses de nitrogênio $(N)$ e fósforo $(P)$ no teor de $N$ nas folhas (TNF), teor de $N$ no caule (TNC), teor de $N$ nas raízes (TNR), teor de $\mathrm{P}$ nas folhas (TPF), teor de $\mathrm{P}$ no caule (TPC) e teor de $\mathrm{P}$ nas raízes (TPR) de mudas de mogno-africano.

\begin{tabular}{|c|c|c|c|c|c|c|c|}
\hline $\mathbf{N}$ & $\mathbf{P}$ & TNF & TNC & TNR & TPF & TPC & TPR \\
\hline \multicolumn{3}{|c|}{ Doses (mg.dm-3) } & \multicolumn{5}{|c|}{ 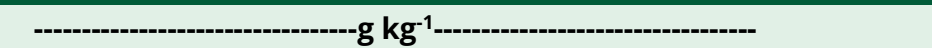 } \\
\hline \multirow{6}{*}{0} & 0 & $18,7 \mathrm{bc}$ & $5,0 a$ & $6,9 a$ & $1,1 a$ & $3,9 b$ & $0,8 a$ \\
\hline & 50 & $22,5 a b$ & $5,0 a$ & $6,6 a$ & $1,1 a$ & $4,7 a b$ & $0,8 a$ \\
\hline & 100 & $16,7 c$ & $5,0 a$ & $7,8 a$ & $1,0 a$ & $4,6 a b$ & $0,8 a$ \\
\hline & 150 & $19,4 a b c$ & $5,0 a$ & $8,1 a$ & $1,2 \mathrm{a}$ & $5,1 \mathrm{ab}$ & $0,9 a$ \\
\hline & 200 & $23,7 a$ & $4,6 a$ & $5,8 a$ & $1,1 a$ & $5,6 a$ & $0,5 a$ \\
\hline & & $F=6,5 ;<0,00$ & $F=0,0 ; p<0,98$ & $F=1,6 ; p<0,21$ & $F=0,7 ; p<0,60$ & $F=4,0 ; p<0,02$ & $\begin{array}{l}F=2,5 ; \\
p<0,08\end{array}$ \\
\hline \multirow{5}{*}{50} & 0 & $24,4 a$ & $7,0 a$ & $7,4 a$ & $0,8 a b$ & $2,7 b$ & $0,8 a$ \\
\hline & 50 & $22,9 a$ & $5,8 a$ & $8,1 a$ & $0,9 a b$ & $3,8 a$ & $0,7 a b$ \\
\hline & 100 & $25,6 a$ & $4,6 a$ & 7,3a & $1,0 a$ & $3,3 a b$ & $1,0 a$ \\
\hline & 150 & $24,1 a$ & $5,0 a$ & $6,2 a$ & $0,7 b$ & $3,1 a b$ & $0,9 a$ \\
\hline & 200 & $31,1 a$ & $5,0 a$ & $7,0 a$ & $0,9 a b$ & $3,7 a$ & $0,3 b$ \\
\hline \multirow{7}{*}{100} & & $F=2,5 ;<0,07$ & $F=2,3 ; p<0,10$ & $F=1,3 ; p<0,29$ & $F=3,1 ; p<0,04$ & $F=5,9 ; p<0,00$ & $\begin{array}{l}F=7,3 ; \\
p<0,00\end{array}$ \\
\hline & 0 & $31,5 a b$ & $17,5 a$ & $9,7 a$ & $1,1 a$ & $1,0 a$ & $0,48 b$ \\
\hline & 50 & $32,6 a b$ & $18,7 a$ & $8,9 a$ & $1,4 a$ & $1,5 a$ & $0,63 a b$ \\
\hline & 100 & $34,6 a$ & $7,4 b$ & $11,2 a$ & $1,5 a$ & $1,4 a$ & $0,67 a b$ \\
\hline & 150 & $26,0 \mathrm{bc}$ & $7,7 b$ & $10,1 a$ & $1,2 a$ & $1,0 a$ & $0,73 a$ \\
\hline & 200 & $23,3 c$ & $5,0 b$ & $10,9 a$ & $1,5 a$ & $1,3 a$ & $0,70 a b$ \\
\hline & & $F=9,6 ;<0,00$ & $F=69 ; p<0,00$ & $F=0,6 ; p<0,64$ & $F=3,9 ; p<0,02$ & $F=1,1 ; p<0,37$ & $\begin{array}{l}F=3,6 \\
p<0,02\end{array}$ \\
\hline \multirow{5}{*}{150} & 0 & $32,3 a$ & $8,2 a b c$ & $8,9 a$ & $1,5 a$ & $0,9 d$ & $0,7 a$ \\
\hline & 50 & $31,5 a$ & $11,6 a$ & $8,5 a$ & $1,8 a$ & $2,4 b$ & $0,7 a$ \\
\hline & 100 & $28,3 a$ & $10,1 a b$ & $8,5 a$ & $1,6 a$ & $3,0 a$ & $0,8 a$ \\
\hline & 150 & $29,9 a$ & $7,4 b c$ & $9,3 a$ & $1,7 a$ & $1,6 c$ & $0,8 a$ \\
\hline & 200 & $28,0 a$ & $5,8 c$ & $8,9 a$ & $1,8 a$ & $2,2 b$ & $0,7 a$ \\
\hline \multirow{7}{*}{200} & & $F=0,9 ; p<0,47$ & $F=7,0 ; p<0,00$ & $F=0,2 ; p<0,88$ & $F=1,4 ; p<0,26$ & $F=50 ; p<0,00$ & $\begin{array}{l}F=0,3 ; \\
p<0,87\end{array}$ \\
\hline & 0 & $30,7 a b$ & $20,2 a$ & $11,6 a b$ & $1,8 a$ & $1,4 b$ & $0,7 a b c$ \\
\hline & 50 & $37,3 a$ & $17,9 a$ & $13,6 a$ & $1,8 a$ & $2,4 a$ & $0,8 a b$ \\
\hline & 100 & $30,0 a b$ & $20,2 a$ & $12,4 a b$ & $2,0 a$ & $1,8 b$ & $0,9 a$ \\
\hline & 150 & $30,0 a b$ & $18,6 a$ & $10,0 b$ & $2,0 a$ & $1,6 b$ & $0,4 c$ \\
\hline & 200 & $28,4 b$ & $8,1 \mathrm{~b}$ & $12,0 a b$ & $1,9 a$ & $0,8 c$ & $0,5 b c$ \\
\hline & & $F=3,1 ; p<0,04$ & $F=19 ; p<0,00$ & $F=2,6 ; p<0,07$ & $F=1,2 ; p<0,34$ & $F=32 ; p<0,00$ & $\begin{array}{l}F=7,6 \\
p<0,00\end{array}$ \\
\hline
\end{tabular}

Médias seguidas de mesma letra na coluna não diferem significativamente.

O TNC das mudas submetidas à $100 \mathrm{mg} \mathrm{dm}^{-3}$ de $\mathrm{N}$ apresentaram decréscimo mediante o aumento das doses de $\mathrm{P}$ (Tabela 3). As mudas cultivadas na ausência de P, apresentaram valor médio de $17,5{\mathrm{~g} . \mathrm{kg}^{-1}}^{-}$, cerca de $243 \%$ superior à média do teor observado $\left(5,1 \mathrm{~g} \mathrm{~kg}^{-1}\right)$ nos indivíduos que receberam a dose máxima (200 mg. $\mathrm{dm}^{-3}$ de P) (Tabela 3). Observou-se acréscimo no TPC das mudas que não receberam adubação nitrogenada $\left(0 \mathrm{mg} . \mathrm{dm}^{-3} \mathrm{~N}\right) \mathrm{em}$ função do aumento das doses de P. Para as mudas cultivadas na dose máxima de $\mathrm{P}\left(200 \mathrm{mg} \cdot \mathrm{dm}^{-3}\right)$, o teor médio foi de $5,6 \mathrm{~g} \cdot \mathrm{kg}^{-1}$ de $\mathrm{N}$, o qual foi $43,6 \%$ superior à média observada $\left(3,9 \mathrm{~g} \cdot \mathrm{kg}^{-1}\right)$ nas mudas cultivadas na ausência de $\mathrm{P}\left(0 \mathrm{mg} \cdot \mathrm{dm}^{-3}\right)$ (Tabela 3).

Os TPR das mudas cultivadas nas doses 100 e $150 \mathrm{mg} \mathrm{dm}^{-3}$ de $\mathrm{N}$ também apresentaram acréscimo nos valores em função do aumento das doses de $\mathrm{P}$. As mudas cultivadas na dose $100 \mathrm{mg} \cdot \mathrm{dm}^{-3}$ de $\mathrm{N}$ e na dose máxima de $\mathrm{P}\left(200 \mathrm{mg} \cdot \mathrm{dm}^{-3}\right)$ apresentaram teor médio de $0,7 \mathrm{~g} \cdot \mathrm{kg}^{-1}$, valor $40 \%$ superior à média observada $\left(0,5 \mathrm{~g} \cdot \mathrm{kg}^{-1}\right)$ para as mudas que não receberam $P$. As mudas cultivadas na dose $150 \mathrm{mg}^{-\mathrm{dm}^{-3}}$ de $\mathrm{N}$ e na dose máxima de $\mathrm{P}$ (200 mg. $\mathrm{dm}^{-3}$ ) 
apresentaram valor médio de $0,8 \mathrm{~g} \cdot \mathrm{kg}^{-1}, 14 \%$ superior à média das mudas $\left(0,7 \mathrm{~g} \cdot \mathrm{kg}^{-1}\right)$ que não receberam fertilização fosfatada $\left(0 \mathrm{mg}^{-\mathrm{dm}^{-3}}\right)$ (Tabela 3 ).

Observa-se, nos ajustes de regressão, efeito significativo entre as diferentes doses de P para as variáveis TNC, quando submetidas a $100 \mathrm{mg}^{-\mathrm{dm}^{-3}}$ de $\mathrm{N}$, para TPC, na dose 0 de $\mathrm{mg}^{\mathrm{dm}} \mathrm{dm}^{-3}$ de N, e TPR, quando submetidas às doses 100 e $150 \mathrm{mg}^{-\mathrm{dm}^{-3}}$ de N (Figura 2B, E e F). As demais variáveis: TNF, TNR e TPF, não apresentaram ajuste de regressão significativo entre as doses de $P$ em nenhuma das doses de $N$ (Figura 2A, C e D).
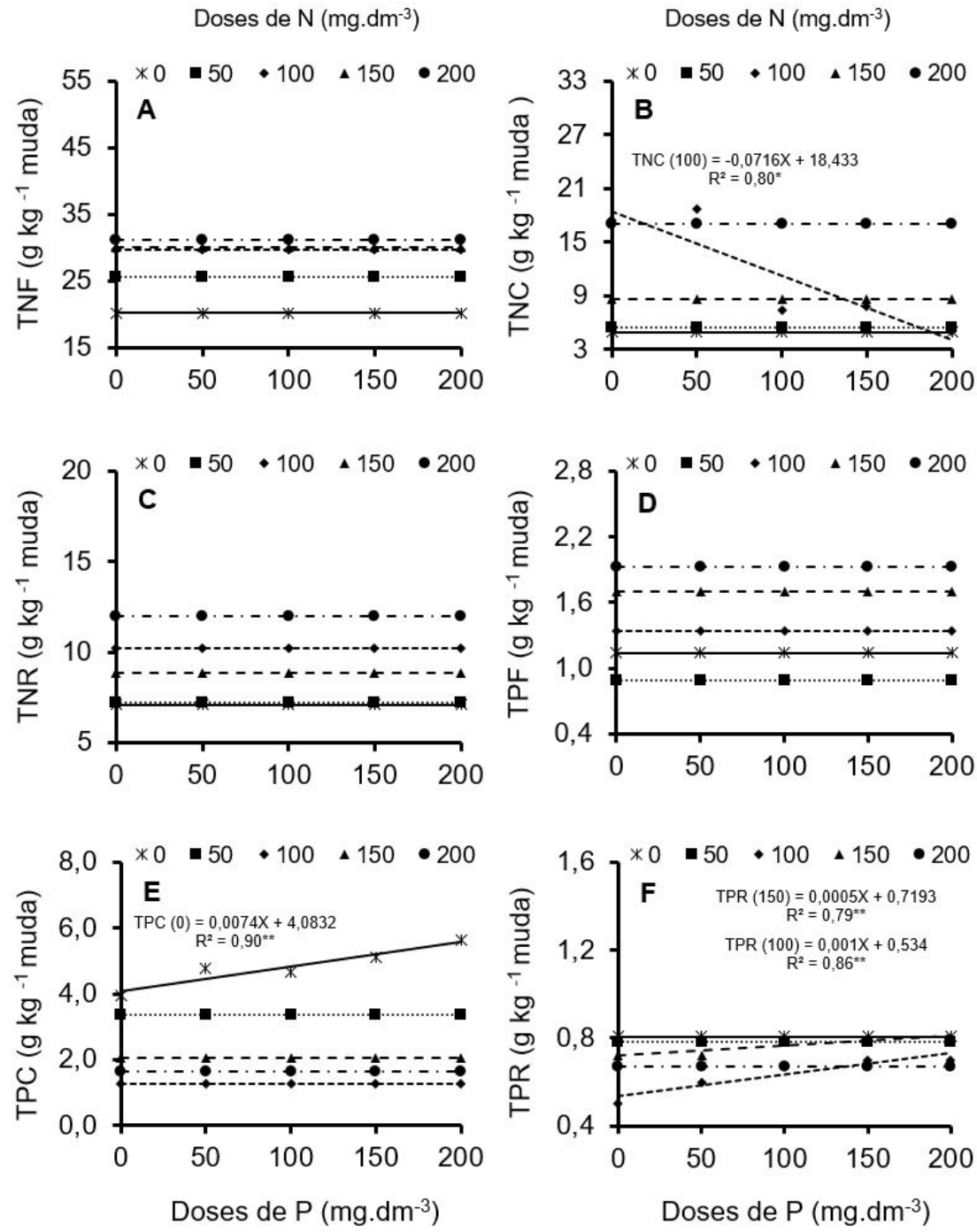

Figura 2. Resultado da análise de regressão para teor de $N$ nas folhas (TNF) (A), no caule (TNC) (B) e na raiz (TNR) (C) e, teor de $P$ nas folhas (TPF) (D), no caule (TPC) (E) e na raiz (TPR) (F) de mudas de mogno-africano em função de doses de $\mathrm{N}$ e P.

\section{DISCUSSÃO}

\section{Características morfológicas}

$\mathrm{O}$ efeito do $\mathrm{N}$ e $\mathrm{P}$ nas mudas de mogno-africano evidenciam a importância da adubação nitrogenada e fosfatada no desenvolvimento inicial desta espécie. É importante salientar que o substrato utilizado apresentava baixo teor de matéria orgânica $\left(7,0 \mathrm{~g} . \mathrm{dm}^{-3}\right)$ e muito baixo teor de $\mathrm{P}\left(0,8 \mathrm{mg} \cdot \mathrm{dm}^{-3}\right)$. O P possui papel crucial no metabolismo das plantas, pois participa de estruturas energéticas que fomentam as atividades celulares e fotossintéticas e também propiciam o desenvolvimento das plantas (Taiz \& Zeiger, 2013; Marschner, 2012). Já o $\mathrm{N}$ no solo encontra-se principalmente na forma orgânica e, em solos com baixo teor de 
matéria orgânica, seu fornecimento via fertilização é essencial, uma vez que este nutriente possui funções estruturais em aminoácidos, proteínas, pigmentos e, principalmente, participa dos processos de fotossíntese, respiração, multiplicação e diferenciação celular (Marschner, 2012).

$\mathrm{O}$ fornecimento de $\mathrm{P}$ na presença de $\mathrm{N}$ também favoreceu o crescimento das raízes, resultando no aumento da massa de matéria seca das raízes das mudas de mogno-africano. Fator que, possivelmente, permitiu a formação de um sistema radicular mais longo e com raízes mais finas, propiciando às plantas melhor exploração de maior volume de solo, resultando na maior absorção de água e nutrientes (Knapik \& Angelo, 2007). Maior desenvolvimento radicular pode ter levado ao maior crescimento e desenvolvimento da altura, diâmetro e massa de matéria seca da parte aérea. A boa formação do sistema radicular, do crescimento em diâmetro e altura das mudas é extremamente importante para a adaptação e desenvolvimento quando submetidas a condições de campo (Ryan et al., 2010).

As mudas de mogno-africano cultivadas sem adição de $\mathrm{P}$ (dose $0 \mathrm{mg} \cdot \mathrm{dm}^{-3}$ ) apresentaram menores médias de massa de matéria seca. Resultados que corroboram com os encontrados por Silva et al. (2009), onde constataram redução de $68 \%$ na produção de matéria seca total de plantas de pinhão-manso (Jatropha curcas L.), quando as plantas foram submetidas à omissão de $P$, assim podemos inferir que as respectivas espécies são exigentes ao fósforo, pois sua falta acarretou sintomas de deficiência do nutriente como a redução no seu crescimento e, consequentemente, menor massa de matéria seca aérea e radicular.

$\mathrm{O} \mathrm{N}$ também afetou de forma positiva o crescimento das características morfológicas (H, DC, MSPA, MSR, MST) das plantas de mogno-africano. Assim como o P, a importância do $\mathrm{N}$ no desenvolvimento inicial desta espécie destaca-se, uma vez que houve incremento nos valores das variáveis analisadas na presença de N. Como o N é necessário para a síntese da clorofila e está envolvido no processo da fotossíntese, em situações de deficiência proporciona menor síntese de clorofila (Reis et al., 2006). Esta situação não permite que a planta utilize a luz solar como fonte de energia no processo fotossintético; deste modo, a planta perde a habilidade de executar funções essenciais, como, por exemplo, a absorção de nutrientes prejudicando o seu desenvolvimento (Novais et al., 2007).

Assim como observado no presente estudo, avaliando o efeito da adubação nitrogenada na produção de mudas de Ipê-amarelo (Tabebuia serratifolia (Vahl) G.Nicholson), Goulart et al. (2017) verificaram acréscimo em altura, diâmetro do coleto, matéria seca da parte aérea, de raiz e total a partir da primeira dose de $\mathrm{N}\left(75 \mathrm{mg} . \mathrm{dm}^{-3}\right)$. Também Tosta et al. (2017), em estudo com Pitomba (Talisia esculenta (A. St. Hil) Radlk), afirmaram que as doses crescentes de $\mathrm{N}$ influenciaram de forma positiva a altura e diâmetro do caule das plantas. Dessa forma, assim como o $\mathrm{P}$, podemos verificar a importância do $\mathrm{N}$ no desenvolvimento inicial de diversas espécies florestais, sendo fundamental o seu fornecimento na fase inicial de crescimento.

É importante mencionar que as plantas de mogno-africano cultivadas na dose $0 \mathrm{mg} \cdot \mathrm{dm}^{-3}$ apresentaram sintomas visíveis de deficiência de $\mathrm{N}$ e $\mathrm{P}$, caracterizados por amarelecimento uniforme generalizado nas folhas mais velhas, reduzida área foliar e baixo crescimento (Novais et al., 2007). Deve-se ressaltar também que, o sintoma visual de deficiência é o último passo de uma série de problemas metabólicos irreversíveis e, quando aparecem, em termos gerais, o dano à muda já foi causado. Por isso, é de extrema importância o fornecimento adequado de $\mathrm{N}$ e $\mathrm{P}$, sendo estas repostas genótipo-dependentes, desta maneira, a dose ótima de um nutriente para determinada espécie pode promover o decréscimo no crescimento de outras.

\section{Teor de nutrientes nas mudas}

Com exceção do TPC e do TNR, menores teores de $\mathrm{N}$ e $\mathrm{P}$ foram detectados no tratamento controle $\left(0 \mathrm{mg} \cdot \mathrm{dm}^{-3}\right.$ de $\mathrm{N}$ e $\left.\mathrm{P}\right)$. $\mathrm{O}$ aumento dos teores de $\mathrm{P}$ e $\mathrm{N}$ nos compartimentos analisados neste trabalho, mediante o fornecimento de níveis crescentes desses nutrientes, possivelmente está relacionado à sua baixa disponibilidade natural no solo estudado [baixo teor de matéria orgânica $\left(7,0 \mathrm{~g} \cdot \mathrm{dm}^{-3}\right)$ e deficiência em $\left.\mathrm{P}\left(0,8 \mathrm{mg} \cdot \mathrm{dm}^{-3}\right)\right] \mathrm{e}$ também à necessidade intrínseca da espécie. Novais et al. (2007) afirmam que em situações 
onde se aumenta o suprimento de $P$, em solos deficientes até adequada disponibilidade para a planta, verifica-se que os teores de $P$ contidos em órgãos vegetativos destas também aumentam, como verificado no TPC do presente trabalho.

Os menores TPC e TPR detectados com a aplicação da dose máxima de N e P decorrem, provavelmente, do efeito de diluição promovido pelo crescimento das plantas, uma vez que as plantas submetidas às doses crescentes $\left(50,100,150\right.$ e $200 \mathrm{mg}^{-\mathrm{dm}^{-3}}$ ) apresentaram maiores crescimentos em todas as variáveis analisadas quando comparadas às plantas do tratamento controle $\left(0 \mathrm{mg} \mathrm{dm}^{-3}\right)$. Resultados semelhantes foram obtidos por Santos et al. (2008) que observaram aumento no teor de P em mudas de mogno cultivadas em Latossolo Amarelo distrófico, em condições de casa de vegetação, em função do fornecimento de doses crescentes de P no solo, apresentando resposta linear no teor de $\mathrm{P}$ até a dose $800 \mathrm{mg}$. $\mathrm{dm}^{-3}$.

Os resultados obtidos nos tratamentos que não receberam adubação nitrogenada e fosfatada reforçam a importância do $\mathrm{N}$ e do $\mathrm{P}$ para o desenvolvimento inicial de mudas de mogno-africano. Em estudo com doses de N e P em mudas de café, Santinato et al. (2014) observaram que na presença de $\mathrm{N}$ houve efeito sinérgico para o aumento linear do teor de $\mathrm{P}$ na planta, apresentando maior teor de $\mathrm{P}$ nas plantas submetidas à adubação nitrogenada, quando comparadas às plantas na ausência de N. Os autores ainda afirmam que, para o teor de $\mathrm{N}$, houve também interação positiva entre os nutrientes, porque com o aumento de doses de $\mathrm{P}$ na presença de $\mathrm{N}$, ocorreu aumento linear no teor do nutriente, fato que não foi observado na ausência de $N$, assim como observado nas mudas de mogno-africano avaliadas no presente trabalho.

De maneira geral, para o crescimento normal das plantas, os teores adequados de $\mathrm{N}$ nos

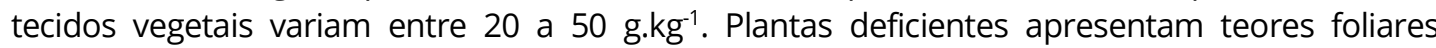
menores do que $10 \mathrm{~g}^{\mathrm{kg}}{ }^{-1}$, enquanto que, acima de $50 \mathrm{~g} \mathrm{~kg}^{-1}$, podem ser observados sintomas de toxidez. Os teores de $\mathrm{P}$ adequados para o crescimento normal das plantas variam de 1,0 a 1,5 g. kg-1. Plantas deficientes apresentam teores foliares menores que 1,0 g.kg-1 , enquanto que, acima de 3,0 g.kg-1 , observam-se sintomas de toxidez (Malavolta et al., 1997).

As plantas de mogno-africano apresentaram teores de $\mathrm{N}$ foliar de $20,2 \mathrm{~g}^{\mathrm{kg}}{ }^{-1}$, na dose $0 \mathrm{mg} \cdot \mathrm{dm}^{-3}$ de $\mathrm{N}$, e $31,3 \mathrm{~g} \cdot \mathrm{kg}^{-1}$ na dose máxima de $\mathrm{N}\left(200 \mathrm{mg}^{-\mathrm{dm}^{-3}}\right)$. Esses teores estão próximos aos encontrados por Almeida et al. (2016) em mudas de pitaia (Hylocereus polyrhizus (F.A.C.Weber) Britton \& Rose). Esses autores verificaram que os teores de $\mathrm{N}$ nas mudas que apresentaram a máxima produção de massa de matéria seca estavam entre 33,7 e 36,9 g. $\mathrm{kg}^{-1}$. Com base nas informações citadas por Novais et al. (2007), os teores encontrados no mogno-africano são adequados tanto para as plantas que não receberam adubação nitrogenada $\left(0 \mathrm{mg} \cdot \mathrm{dm}^{-3}\right)$ quanto para as plantas cultivadas na dose máxima de N (200 mg. $\left.\mathrm{dm}^{-3}\right)$.

No entanto, os resultados permitem afirmar que o nível de exigência de $\mathrm{N}$ da espécie estudada é maior em relação às demais culturas, uma vez que as plantas submetidas à dose $0 \mathrm{mg} \cdot \mathrm{dm}^{-3}$ de $\mathrm{N}$ apresentaram sintomas de deficiência e/ou baixo desenvolvimento nas características morfológicas. Segundo Malavolta et al. (1997), plantas com baixo crescimento tendem a acumular maiores teores de $\mathrm{N}$ no vacúolo, por isso, as plantas de mogno-africano apresentaram valores adequados na análise.

Os teores de $\mathrm{P}$ foliar nas mudas de mogno-africano também podem ser considerados adequados, com 1,3 g. $\mathrm{kg}^{-1}$ nas plantas cultivadas na ausência de $\mathrm{P}$ e 1,5 g.kg-1 nas plantas cultivadas na dose máxima de $\mathrm{P}\left(200 \mathrm{mg}^{\mathrm{dm}} \mathrm{dm}^{-3}\right.$. Contudo, assim como foi observado deficiência de $N$ também foram visualizados sintomas de deficiência de $P$ nas mudas cultivadas na dose $0 \mathrm{mg} \cdot \mathrm{dm}^{-3}$ do nutriente. Deste modo, podemos inferir que a espécie é também exigente em $\mathrm{P}$ na fase inicial de desenvolvimento.

\section{CONCLUSÃO}

As características morfológicas de crescimento (altura, diâmetro do coleto, número de folíolos, massa de matéria seca da parte aérea, de raízes e total) das mudas de mogno-africano foram influenciadas positivamente pela adubação nitrogenada e fosfatada, mostrando-se a exigência destes nutrientes na fase inicial da espécie. 
Recomenda-se para a produção de mudas de mogno-africano (Khaya ivorenses A Chev.)

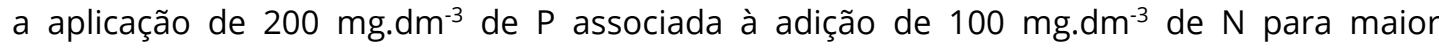
desenvolvimento inicial das mudas.

\section{REFERÊNCIAS BIBLIOGRÁFICAS}

Almeida, E. I. B., Deus, J. A. L. D., Correa, M. C. M., Crisostomo, L. A., \& Neves, J. C. L. (2016). Linha de fronteira e chance matemática na determinação do estado nutricional de pitaia. Ciência Agronômica, 47(4), 744-754.

Araújo, M. S., Cunha, S. D., D’Abadia, K. L., Morais, Y. C. R., Rocha, E. C., Barretto, V. C. M., \& Coelho, G. M. (2017). Initial growth of African mahogany plants in response to zinc fertilization. African Journal of Agricultural Research, 12(12), 1022-1026.

Bissani, C. A., Gianello, C., Tedesco, M. J., \& Camargo, F. A. O. (2004). Fertilidade do solo e manejo da adubação de culturas (328 p.). Porto Alegre: Editora Gênesis.

Cabral, C. E. A., Cabral, L. S., Silva, E. M. B., Carvalho, K. S. C., Kroth, B. E., \& Cabral, C. H. A. (2016). Resposta da Brachiaria brizantha cv. Marandu a fertilizantes nitrogenados associados ao fosfato natural reativo. Comunicata Scientiae, 7(1), 66-72.

Cardoso, A. A. S., Santos, J. Z. L., Tucci, C. A. F., Farias, E. P., \& Moura, R. P. M. (2015). Influência da acidez e do teor de fósforo do solo no crescimento inicial do mogno. Pesquisa Florestal Brasileira, 35(81), 110.

Danquah, A. J., Appiah, M., \& Ari, P. (2011). Eco-geographic variation in leaf morphology of African mohogany (Khaya anthotheca and Khaya ivorensis). European Journal of Scientific Research, 51(1), 18 28.

Empresa Brasileira de Pesquisa Agropecuária - EMBRAPA. (2013). Sistema brasileiro de classificação de solos (3. ed., 201 p.). Brasília: Embrapa Solos.

Goulart, L. M. L., Paiva, H. N., Leite, G. L., Xavier, A., \& Duarte, M. L. (2017). Produção de mudas de ipêamarelo (Tabebuia serratifolia) em resposta a fertilização nitrogenada. Floresta e Ambiente, 24(1), 1-9.

Knapik, J. G., \& Angelo, A. C. (2007). Pó de basalto e esterco eqüino na produção de mudas de Prunus sellowii Koehne (rosaceae). Floresta, 37(3), 427-436.

Leal, A. J. F., Lazarini, E., Rodrigues, L. R., \& Marcandalli, L. H. (2013). Plantas de cobertura e modos de aplicação de calcário. Revista Brasileira de Ciência do Solo, 37, 491-501.

Malavolta, E., Vitti, G. C., \& Oliveira, S. A. (1997). Avaliação do estado nutricional das plantas: princípios e aplicações (2. ed., 319 p.). Piracicaba: POTAFOS.

Marschner, P. (2012). Mineral nutrition of higher plants (3rd ed., 655 p.). London: Academic Press.

Novais, R. F., Alvarez, V. V. H., Barros, N. F., Fontes, R. L. F., Cantarutti, R. B., \& Neves, J. C. L. (2007). Fertilidade do solo (1017 p.). Viçosa: SBCS.

Pinheiro, A. L., Couto, L., Pinheiro, D. T., \& Brunetta, J. M. F. (2011). Ecologia, silvicultura e tecnologia de utilização dos mognos-africanos (Khaya spp.). (102 p.). Viçosa, MG: Sociedade Brasileira de Agrossilvicultura.

Raij, B. V., Andrade, J. C., Cantarella, H., \& Quaggio, J. A. (2001). Análise química para avaliação da fertilidade de solos tropicais (285 p.). Campinas: Instituto Agronômico.

Reis, A. R., Furlani Junior, E., Buzetti, S., \& Andreotti, M. (2006). Diagnóstico da exigência do cafeeiro em nitrogênio pela utilização do medidor portátil de clorofila. Bragantia, 65(1), 163-171.

Ribeiro, A. C., Guimarães, P. T. G., \& Alvarez, V. A. H. (1999). Recomendação para uso de corretivos e fertilizantes em Mina Gerais - $5^{\circ}$ aproximação (359 p.). Viçosa: CFSEMG.

Ribeiro, A., Ferraz Filho, C. A., \& Scolforo, J. R. S. (2017). O cultivo do mogno africano (Khaya spp.) e o crescimento da atividade no Brasil. Floresta e Ambiente, 24(1), 1-11.

Ryan, G. M., Stape, J. L., Binkley, D., Fonseca, S., Loos, A. R., Takahashi, E. N., Silva, C. R., Silva, S. R., Hakamada, R. E., Ferreira, J. M., Lima, A. M. N., Gava, J. L., Leite, F. P., Andrade, H. B., Alves, J. M., \& Silva, G. C. (2010). Factors controlling Eucalyptus productivity: how water availability and stand structure alter production and carbon allocation. Forest Ecology and Management, 259(9), 1695-1703.

Santinato, F., Caione, G., Tavares, T. O., \& Prado, R. M. (2014). Doses of phosphorus associated with nitrogen on development of coffee seedlings. Coffee Science, 9(3), 419-426.

Santos, J. Z. L., \& Resende, A. V., Furtini Neto, A. E., \& Corte, E. F. (2008). Crescimento, acúmulo de fósforo e frações fosfatadas em mudas de sete espécies arbóreas nativas. Revista Árvore, 32(5), 799-807. 
Silva, E. B., Tanure, L. P. T., Santos, S. R., \& Resende Júnior, P. S. (2009). Sintomas visuais de deficiências nutricionais em pinhão-manso. Pesquisa Agropecuária Brasileira, 44(4), 392-397.

Silva, F. C. (2009). Análise química de tecido vegetal. In F. C. Silva (Ed.), Manual de análises químicas de solo, plantas e fertilizantes (2. ed., pp. 193-204). Brasília: Embrapa Informação Tecnológica.

Silva, J. G. M., Vidaurre, G. B., Arantes, M. D. C., Batista, D. C., Soranso, D. R., \& Billo, D. F. (2016). Qualidade da madeira de mogno africano para a produção de serrados. Scientia Forestalis, 44(109), 181-190.

Taiz, L., \& Zeiger, E. (2013). Fisiologia vegetal (5. ed., 954 p.). Porto Alegre: Artmed.

Tosta, M. S., Almeida, J. P. N., Góes, G. B., Freire, P. A., \& Mendonça, V. (2017). Nitrogen fertilization in the production of seedlings of Talisia esculenta (A. St. Hil) Radlk. Revista Brasileira de Engenharia Agrícola e Ambiental, 21(7), 443-447.

Verzignassi, J. R., Poltronieri, L. S., \& Benchimol, R. L. (2009). Mancha-alvo em mogno-africano no Brasil. Summa Phytopathologica, 35(1), 70-71.

Contribuição dos Autores: MSA: conceituação, curadoria de dados, investigação, metodologia, software, administração do projeto, escrita - primeira redação, escrita - revisão e edição; SDC: análise formal, curadoria de dados, visualização, escrita - revisão e edição; AG: conceituação, análise formal, metodologia, recursos, supervisão, validação; BERH e AP: conceituação, análise formal, metodologia, recursos, supervisão, validação; escrita - revisão e edição. 